\title{
Estudio comparativo de la gravedad atribuida a las conductas disruptivas en el aula
}

\section{Concepción Gotzens Busquets, Mar Badia Martín, Cándido Genovard Rosselló, Teresa Dezcallar Sáez}

Facultad de Psicología, Universidad Autónoma de Barcelona

\section{España}

Correspondencia: Concepción Gotzens Busquets. Dpto. de Psicología Básica, Evolutiva y de la Educación. Facultad de Psicología.Campus de Bellaterra. Edificio B. Universidad Autónoma de Barcelona. Bellaterra (Barcelona). España. E-mail: concepcio.gotzens@uab.es

Este artículo se ha desarrollado en el marco del Proyecto I+D+ I subvencionado por el Ministerio de Educación y Ciencia con código SEJ2005-02688

(C) Education \& Psychology I+D+i and Editorial EOS (Spain) 


\section{Resumen}

Introducción. En este artículo presentamos un análisis comparativo de tres muestras de docentes de Coimbra, Barcelona y Murcia que proporciona una visión sobre la importancia que conceden los docentes a las conductas disruptivas y cómo las diversas perspectivas y valoraciones afectan el quehacer diario del profesorado.

Método. Esta investigación se enmarca en un diseño cuantitativo empírico-analítico. Se trata de un estudio descriptivo que compara los datos obtenidos a partir de la aplicación de un cuestionario a una muestra de 146 docentes de enseñanza infantil, primaria y secundaria, de tres ciudades europeas: Barcelona, Murcia, y Coimbra. El cuestionario, de tipo no obtrusivo, ha sido elaborado por las autoras a fin de minimizar las distorsiones asociadas al lenguaje.

Resultados. Los resultados obtenidos destacan sistemáticamente la tendencia propuesta en las hipótesis del estudio. En general, las conductas sociales tienden a obtener puntuaciones más elevadas que las conductas instruccionales, exceptuando el caso de desobedecer que obtiene la máxima puntuación. Los profesores tienden a atribuir mayor puntuación a las conductas instruccionales que sus colegas del sexo femenino y se observa una tendencia a atribuir mayor gravedad a los comportamientos instruccionales a medida que aumenta los años de experiencia docente.

Discusión y Conclusión. El profesorado de este estudio muestra mayor preocupación por las transgresiones de orden social general que se dan en el grupo de clase, relegando a posiciones de menor importancia las conductas disruptivas de tipo instruccionales cuya implicación en los procesos de enseñanza-aprendizaje está rigurosamente comprobada. Por otra parte, los años de experiencia parecen ejercer un efecto moderador en la preocupación por las conductas sociales llegándolas a equiparar en gravedad con las instruccionales, observándose dicha equiparación de forma preferente entre los docentes del sexo masculino que entre sus compañeras del sexo femenino.

Palabras Clave: Disciplina en el aula, estudio comparativo, percepción docentes, experiencia docente.

Recibido: 21/09/09 Aceptación Inicial: 22/09/09 Aceptación Definitiva: 25/11/09 


\title{
A comparative study of the seriousness attributed to disruptive classroom behaviors
}

\begin{abstract}
Introduction. In this paper we present a comparative analysis of three samples of teachers from Coimbra, Barcelona and Murcia that provides insight into the importance attached by teachers to disruptive behaviour and how different perspectives and values affect their daily work.
\end{abstract}

Method. This research is part of a quantitative empirical-analytic design. This is a descriptive study that compares the data obtained from the application of a questionnaire to a sample of 146 school teachers of primary and secondary education in three European cities: Barcelona, Murcia and Coimbra. The questionnaire, an unobtrusive type, has been developed by the authors to minimize the distortions associated with language.

Results. The results highlight the trend consistently proposed by the study hypothesis. In general, social behaviours have to score higher than instructional behaviours, except in the case of disobedience that gets the high score. Teachers tend to attribute higher scores to the behaviour of instructional female colleagues and a tendency to attribute most seriously instructional behaviours with increasing years of teaching experience.

Discussion and Conclusion. The teachers sampled in this study show greater concern for the general social transgressions that occur in the class group, relegating to minor positions of disruptive behaviours, such as involvement in instructional teaching-learning processes which are rigorously proven. Moreover, years of experience appear to exert a moderating effect on the concern for social behaviours to the extend to equate the same gravity with the instructional processes observed. Such parity is observed between male teachers and among their female partners as well.

Keywords: Classroom discipline, comparative study, teachers' perceptions, teaching experience.

Received: 09/21/09 Initial Acceptance: 09/22/09 Definitive Acceptance: 11/25/09 


\section{Introducción}

Los problemas de disciplina en el ámbito educativo resultan comunes en todas las áreas y asignaturas que constituyen el currículo de educación (Hardman y Smith, 2003; Ishee, 2004), con serias repercusiones en el proceso de enseñanza-aprendizaje ya que, entre otras razones, limitan el tiempo de dedicación al aprendizaje del alumno (Fernández-Balboa, 1990). Aproximadamente, la mitad del tiempo en el aula se consume en actividades que no tienen que ver con los contenidos instruccionales y sí con los problemas de disciplina (Bibou, Kiosseoglou y Stogiannidou, 2000). Del mismo modo, los comportamientos indisciplinados del alumnado, a menudo, representan una fuente de estrés profesional, así como de cuestionamiento de la labor desempeñada por parte del profesor, generando ansiedad, preocupación e incluso abandono de la profesión (Fernández-Balboa, 1991), especialmente en el profesorado noveles (Borko, Lalik y Tomchin, 1987).

No es difícil encontrar información en la prensa que señala la ausencia de disciplina como el problema más grave al que se enfrenta el sistema de educativo. Muchos profesores y estudiantes están preocupados acerca del desorden y riesgo de mal ambiente en las aulas y en los centros escolares. El personal escolar docente y no docente, los estudiantes, y los padres llaman la atención sobre la elevada incidencia de problemas de comportamiento que se dan en la escuela -consumo de drogas, engaño, insubordinación, absentismo, intimidación, etc.- con graves repercusiones en la comunidad educativa.

Pero es importante señalar que, además de estas conductas de naturaleza social que alteran la dinámica y el orden en el aula (Meunier, 2000; Ramírez y Justicia, 2006), existe otro tipo de conductas disruptivas que también interrumpe el flujo de actividades del aula e imposibilita el aprendizaje del alumnado; son las denominadas conductas disruptivas instruccionales (Gotzens, 1997; Grebennikov, 2005). Dichas conductas se caracterizan por alterar el orden en el aula a consecuencia de infringir o desconocer las normas específicas que regulan el funcionamiento del grupo de clase, distintas a las que presiden el funcionamiento de otros grupos.

Ello puede suceder bien porque el docente no ha elaborado suficientemente la regulación de la disciplina del aula, bien porque no la ha dado a conocer al alumnado o bien porque estos -o algunos de ellos- la infringen (Corrie, 1997). En todo caso se trata de conductas disruptivas que el docente puede gestionar y optimizar, es decir, que está en su mano el preverlas 
o, en todo caso, intervenir de manera directa sobre las mismas (Gotzens, Badia, Castelló y Genovard, 2007).

Valoración de los comportamientos disruptivos a lo largo del S. XX

La preocupación por concretar y analizar la problemática de los comportamientos disruptivos se hace patente en los diversos estudios que existen sobre la percepción de profesores, alumnos y padres sobre dicho tema (Houhton, Merrett y Whendall, 1988). En un trabajo anterior (Gotzens, 1985) analizamos las principales investigaciones que hasta los años 80 del S.XX se habían realizado sobre la importancia atribuida por los adultos a los problemas de disciplina escolar, y nos parece interesante revisar algunos aspectos a la luz de lo sucedido en los últimos 25 años.

Con tal propósito retomamos el estudio realizado por Wickman (1928), pionero en este género, que consistió en presentar una lista de 50 comportamientos de indisciplina a 511 maestros y 30 psicólogos clínicos solicitándoles que los ordenasen según el grado de importancia que otorgaban a cada uno de ellos desde la perspectiva de la disciplina escolar. Los resultados mostraron que los profesores se mostraban más preocupados por las transgresiones del orden y la moralidad, y consideraban que el robo, la masturbación, las notas obscenas, la mentira, las faltas a clase y la impertinencia eran los problemas más serios. Dicho en otras palabras, el desafío a la autoridad, a las normas morales y al control del aula eran percibidas como conductas altamente amenazantes.

Por su parte, los psicólogos clínicos señalaban las conductas suicidas como el principal motivo de preocupación en el ámbito de la disciplina escolar. Los ítems que ocupaban los espacios inmediatamente posteriores se referían a conductas asociadas a trastornos de personalidad.

Grosso modo, el estudio de Wickman puso de manifiesto la existencia de ideas dispares sobre el concepto de disciplina escolar: mientras que el profesorado destacaban la mayor capacidad disruptiva de conductas amenazadoras del orden en clase (robo, sexo, engaño, impertinencia, etc.), los psicólogos clínicos consideran que eran los trastornos individuales del alumnado los que poseían mayor potencial dañino en lo que a alteración del orden se refería.

A pesar de que, en la época en que se llevó a cabo el estudio, el tema disciplina escolar carecía de la importancia y las connotaciones que posee en la actualidad, la diferencia se- 
ñalada en el párrafo anterior resulta particularmente interesante en tanto pone de manifiesto desacuerdos y confusiones conceptuales, algunos de los cuales persisten todavía:

En primer lugar, la perspectiva docente y la psicológica difieren en la interpretación de los propósitos y tareas propias de la escuela: mientras que los docentes parecen necesitar una situación de orden para llevar a cabo las tareas de enseñar que tienen conferidas a fin de promover el aprendizaje del alumnado, los psicólogos ponen el acento en la necesidad de lograr y mantener el equilibrio personal de estos.

En segundo lugar, las problemáticas destacadas por los profesores se refieren a alteraciones del orden llevadas a cabo por alumnos individuales o por grupos de alumnos pero que, en su mayoría, representan una perturbación del orden general de la clase, en tanto las preocupaciones de los psicólogos se centran en casos individuales en los que nace y muere la problemática, sin prestar excesiva atención a las repercusiones que de ello se pudieran derivar para el resto del grupo de alumnos.

En tercer lugar, en tanto que los docentes parecen mostrarse más preocupados por conductas de frecuente aparición en el aula, aunque de moderada gravedad intrínseca, los psicólogos se sitúan en la perspectiva opuesta donde lo más acuciante es la gravedad del comportamiento frente a su -por fortuna- escasa frecuencia de presentación.

No será hasta la segunda mitad del s. XX, y por motivos que se relacionan con el desarrollo de las diversas disciplinas que se matizarán algunas de las diferencias mencionadas. Básicamente, en el ámbito escolar los problemas clínicos se minimizarán adquiriendo preponderancia el análisis e intervención psicoeducativa en lugar de clínica (Gotzens, 2006; Watkins y Wagner, 1991; White, Algozzine, Audette, Marr y Ellis, 2001). Ello no significa que los psicólogos clínicos abandonen la escuela, sino que los psicólogos de la educación se aproximan al estudio de los procesos de enseñanza-aprendizaje, propios de dicho ámbito, logrando detectar mejor los problemas que en surgen en el aula y en la escuela, consiguiendo así una mayor sintonía con las preocupaciones de los docentes.

El interés primigenio de la psicología por los individuos, repercutirá largamente en la investigación sobre los procesos de enseñanza-aprendizaje en tanto que los que se llevan a cabo en la escuela, no se desarrollan de manera individual sino en el seno de grupos (Kounin, 1977). Esta cuestión, aun siendo fundamental para la comprensión de los procesos citados, ha 
sido ignorada con frecuencia por los propios psicólogos de la educación tendiendo a reproducir el esquema terapeuta-paciente en su análisis de las relaciones profesor-alumno.

Ello ha conllevado que, si bien los trastornos de personalidad del alumno no constituyen el nucleo de la preocupación psicoeducativa por el tema de la disciplina escolar, ésta aún se plantea frecuentemente desde una perspectiva de casos individuales, lo que obliga tanto al maestro, como al psicólogo, como al personal de apoyo a enfrentarse a casos individuales que, la mayoría de la veces, no hallan solución en el contexto de un grupo de clase.

\section{Planteamientos actuales}

Los planteamientos más actuales sobre el tema surgen de dos consideraciones fundamentales. Por una parte, la disciplina en el aula no es un problema individual sino colectivo, ya que el grupo de clase no es una suma de individuos sino un conjunto de personas cuyas actividades suponen numerosas y múltiples interacciones. Por ello la disciplina escolar no puede focalizarse en un caso individual, sino que debe atender al buen funcionamiento del grupo clase (Gump, 1980).

De ahí que el concepto interacción represente un claro avance en el planteamiento del tema que nos ocupa (Genovard y Gotzens, 1990; Richmond y McCroskey, 1992; Slavin, 1989). Sin embargo, dado que la interacción ocurre en todos grupos humanos, se observa cierta tendencia a extrapolar sus características de un contexto a otro ignorando las peculiaridades propias de cada uno. Así, por ejemplo, las ideas asociadas a las normas en la interacción en el contexto familiar se han aplicado igualmente al contexto escolar, generando una confusión de efectos nada despreciables.

De manera que algunas faltas que en el contexto familia o en el contexto vecindad producen graves alteraciones, pueden resultar menos disruptivas en el contexto escolar y viceversa, según la infracción altere el orden necesario para que cada uno de estos contexto funcione y pueda acceder a los propósitos encomendados.

En lo referente a la segunda consideración citada sobre la toma de conciencia de que los procesos instruccionales precisan de condiciones específicas para llevarse a cabo (McLeod, Fisher y Hoover, 2003; Rudduck, Chaplain y Wallace, 1996; Seidman, 2005), lo que plantea la necesidad de establecer normas relacionadas con el funcionamiento del aula y el orden que lo hace posible (por ejemplo: realizar las tareas encomendadas, participar en las activida- 
des de aprendizaje, seguir las instrucciones del profesor, etc.) y no únicamente las que se refieren a cuestiones de orden social general (apropiarse de objetos ajenos, destruir material, acoso, etc.) y que, en términos de enseñanza-aprendizaje, las primeras constituyen auténticas condiciones sine qua non para que el proceso de enseñanza-aprendizaje se produzca con garantías.

Sin embargo, trabajos anteriores sobre el tipo de conocimientos que guían la acción docente (Calderhead, 1996; Castelló, 1999; Mumby, Russell y Martin, 2001; Pajares, 1992; Woolfolf, Rarosoff y Hoy, 1998;) han puesto de manifiesto que ante la falta de proyectos formativos rigurosos, las creencias constituyen una parte irrenunciable del mismo, por lo que el conocimiento formal es remplazado por el de orden creencial, siendo éste último de difícil modificación.

A pesar de que existen algunas experiencias de formación del profesorado sobre temas relacionados con la convivencia en clase integrada en el propio currículum académico (Benítez, García-Berbén y Fernández, 2009), la situación más frecuente en nuestro país es que la formación sobre disciplina escolar esté ausente de los planes de formación de futuros docentes. Por ello, la hipótesis de este trabajo se refiere a que el profesorado tiende a aplicar conocimiento de tipo creencial antes que científico sobre la organización y gestión de la disciplina escolar. Así, la mayoría toma como referencia los comportamientos que la sociedad considera perturbadores e indeseables (conductas sociales) y tienden a extrapolarlos al contexto escolar, menospreciando la importancia de los que son fundamentales para el buen funcionamiento del grupo de clase (conductas instruccionales) (Badia, 2001; Gotzens, Castelló, Genovard y Badia, 2003).

\section{Objetivos e hipótesis}

En el presente trabajo nos proponemos valorar cuáles son las principales preocupaciones del profesorado sobre disciplina en el aula. Concretamente, pretendemos identificar la gravedad que los docentes atribuyen a diversos problemas de comportamiento que se dan en el aula, algunos de los cuales denominamos "problemas sociales" y otros de carácter básicamente "instruccional" pues -como ya hemos comentado más arriba- aunque el contexto escolar pueden ser considerado como una extensión del mundo social, posee unas reglas y condiciones específicas que son distintas a las que rigen en otros sectores del "mundo social".

Cuando existen confusiones entre estas normas y condiciones, el profesorado muestran inconsistencias pues rechazan e incluso castigan comportamientos que, en su propia opinión, 
no consideran merecedores de tal respuesta por parte del docente (Gotzens, Castelló, Genovard, Badia, 2003). Adicionalmente, hemos creído interesante efectuar dicha constatación en muestras de profesorado diversas, tanto en lo que se refiere a zona geográfica, años de experiencia, género, como a nivel de enseñanza impartido. Fruto de estos propósitos, planteamos las hipótesis siguientes:

\section{Hipótesis 1}

Pretendemos verificar que la preocupación del profesorado recae principalmente en la aparición de comportamientos socialmente graves, tales como robar, mentir, etc. aunque no comporten excesivo riesgo en términos de enseñanza-aprendizaje, en lugar de otorgar preferencia a los que comprometen seriamente el desarrollo de estos procesos si bien no necesariamente poseen un riesgo social significativo, como por ejemplo inhibirse de las tareas a realizar olvidar sistemáticamente los materiales escolares en casa, etc. (conductas instruccionales)

\section{Hipótesis 2}

Esta hipótesis se subdivide en cuatro referidas a las diferencias esperadas en las cuatro variables analizadas en los 146 docentes que conforman la muestra. Así,

\section{Subhipótesis 2.1}

Se espera observar diferencias en las valoraciones del profesorado según el género, en el sentido de que las profesoras atribuirán mayor gravedad a los comportamientos sociales frente a los instruccionales que los profesores.

\section{Subhipótesis 2.2}

Se espera observar diferencias en las valoraciones del profesorado según la etapa educativa en la que imparten docencia, en el sentido de que los de las primeras etapas (infantil y primaria) atribuirán mayor gravedad a los comportamientos sociales, mientras que en la etapa de secundaria se atribuirá mayor gravedad a los comportamientos instruccionales.

\section{Subhipótesis 2.3}

Se espera observar diferencias en las valoraciones de los profesores según los años de experiencia acumulados, en el sentido de que los docentes de menor experiencia atribuirán 
mayor gravedad a los comportamientos sociales, mientras que los de mayor experiencia lo harán a los comportamientos instruccionales.

\section{Subhipótesis 2.4}

Finalmente, en relación a la variable población o zona geográfica señalamos que su planteamiento es más exploratorio que de verificación, por lo que no formulamos ninguna expectativa en concreto.

\section{Método}

\section{Participantes}

La muestra está compuesta por 146 profesores de tres ciudades europeas: Barcelona, Murcia y Coimbra. La tabla 1 muestra la distribución de los sujetos según género, etapa educativa, años de experiencia y población. Al igual que en anteriores trabajos ya citados y a causa de ciertos recelos y temores asociados a las declaraciones y toma de postura sobre esta temática, la paticipación de los sujetos de la muestra es anónima y voluntaria. La distribución del profesorado en lo que a género, años de experiencia y etapa educativa se refiere se presenta en la siguiente tabla.

\section{Tabla 1. Distribución de las muestras}

\begin{tabular}{|c|c|c|c|c|}
\hline & & & & \\
\hline & & \multicolumn{3}{|c|}{ POBLACIÓN (N=146) } \\
\hline & & $\begin{array}{l}\text { Barcelona } \\
(\mathrm{N}=46)\end{array}$ & $\begin{array}{c}\text { Coimbra } \\
(\mathrm{N}=66)\end{array}$ & $\begin{array}{l}\text { Murcia } \\
(\mathrm{N}=34)\end{array}$ \\
\hline \multirow{2}{*}{ GÉNERO } & Masculino $(\mathrm{N}=31)$ & 6 & 16 & 9 \\
\hline & Femenino $(\mathrm{N}=115)$ & 40 & 50 & 25 \\
\hline \multirow{3}{*}{$\begin{array}{c}\text { ETAPA } \\
\text { EDUCATIVA }\end{array}$} & Infantil $(\mathrm{N}=26)$ & 19 & 3 & 4 \\
\hline & Primaria $(\mathrm{N}=82)$ & 21 & 36 & 25 \\
\hline & Secundaria $(\mathrm{N}=38)$ & 6 & 27 & 5 \\
\hline \multirow{4}{*}{$\begin{array}{l}\text { AÑOS DE EXPE- } \\
\text { RIENCIA }\end{array}$} & $1-5(\mathrm{~N}=32)$ & 11 & 15 & 6 \\
\hline & $6-10(\mathrm{~N}=34)$ & 13 & 16 & 5 \\
\hline & $11-20(\mathrm{~N}=45)$ & 12 & 21 & 12 \\
\hline & $>20(\mathrm{~N}=35)$ & 10 & 14 & 11 \\
\hline
\end{tabular}

\section{Instrumento y procedimiento}

La información analizada representa las opiniones manifestadas por los docentes sobre la gravedad otorgada a ciertos comportamientos que se presentan en el aula, o lo que es lo 
mismo: el valor potencialmente disruptivo que el profesorado atribuye a diversos problemas de comportamiento.

Dichos valores se han contrastado en función de las variables ya indicadas: años de experiencia, etapa educativa, género y población de los sujetos de la muestra, dando lugar a contrastes inter-grupos. Paralelamente, al comparar las valoraciones otorgadas por los profesores respecto a los comportamientos sociales y a los comportamientos instruccionales, hemos aplicado contrastes intra-grupo.

Dado que en investigaciones anteriores ya habíamos detectado "interferencias" creadas por el lenguaje, en el sentido de que es muy difícil que un profesor afirme, por ejemplo, que "robar a un compañero" no es un comportamiento altamente disruptivo en el aula, optamos por diseñar un instrumento de tipo gráfico y de carácter no obstrusivo con la pretensión de evitar al máximo las distorsiones en las respuestas de los docentes.

Como muestra la figura 1, dicho instrumento consiste en una diana formada por cuatro círculos concéntricos y un listado de ocho comportamientos disruptivos identificados con las ocho primeras letras del alfabeto a fin de evitar el carácter predominantemente jerárquico que suele atribuirse al uso de números.
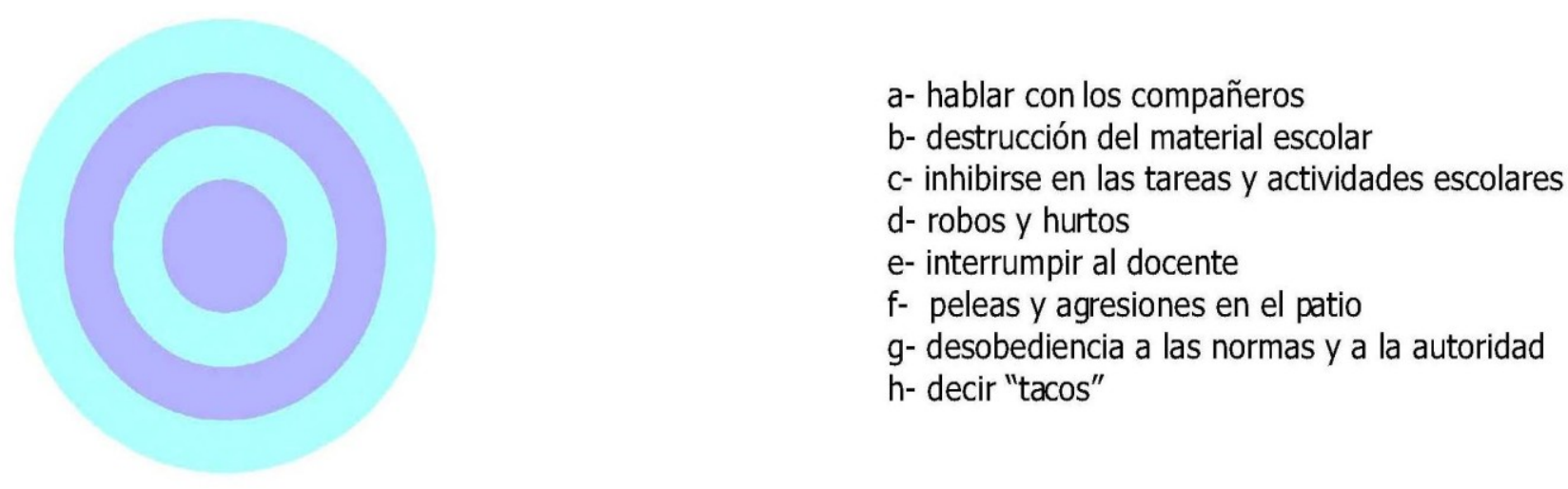

Figura 1. Instrumento elaborado y utilizado en el estudio

De los comportamientos presentados, cuatro de ellos correspondían a la categoría de "comportamientos disruptivos de tipo social" y otros cuatro a la de "comportamientos disruptivos de tipo instruccional". La demanda formulada a los docentes de nuestras muestras de estudio consiste en ubicar las letras correspondientes a cada comportamiento en algún lugar 
de la diana, teniendo en cuenta que cuanto más hacia el centro, mayor gravedad se otorgaba al comportamiento y cuanto más alejado del mismo, menor es la gravedad que se le imputa.

La consigna que se leía al inicio del cuestionario era la siguiente: "Por favor, sitúe en la siguiente diana los 8 comportamientos que aparecen en la columna de la derecha. Se trata de distribuirlos de manera que los que consideres más perturbadores para el buen desarrollo de la clase ocupen los lugares centrales de la diana, mientras que los que le parezcan menos disruptivos ocupen los lugares más periféricos. Si alguno de ellos no le parece perturbador, sitúelo fuera de la diana".

A cada comportamiento se le otorgaron 4 puntos si estaba ubicado en el centro de la diana, 3 en el siguiente y así sucesivamente hasta 0 puntos si se ubicaba fuera del círculo exterior. Así pues, la escala de puntuación tenía un rango efectivo de 4 puntos.

Por motivos que ya hemos comentado en estudios anteriores y cuyas limitaciones y ventajas hemos analizado ampliamente (Añaños y Gotzens, 1990) decidimos garantizar tanto el anonimato de los participantes, por lo que los datos solicitados se refieren exclusivamente a las cuatro variables citadas más arriba, como el carácter voluntario de su colaboración.

Así, tras contactar con los directores de diversos centros escolares de las poblaciones citadas y explicar las características del estudio, se concertó día y hora para la administración de la prueba de carácter anónimo y voluntario. A pesar de no establecer límite de tiempo para la administración de la prueba, ningún caso superó los 10 minutos.

\section{Diseño y Análisis Estadístico}

Esta investigación se enmarca en un diseño cuantitativo empírico-analítico. Se trata de un estudio descriptivo que compara los datos obtenidos a partir de la aplicación de un cuestionario a una muestra de 146 docentes de enseñanza infantil, primaria y secundaria, de tres ciudades europeas: Barcelona, Coimbra y Murcia.

Para el tratamiento estadístico de los datos obtenidos se ha utilizado el software de análisis estadístico SPSS v15.0. Los análisis han consistido en pruebas bivariadas, utilizando el modelo no paramétrico adecuado (Test de Mann-Whitney-Wilcoxon o Test de KruskalWallis). 


\section{Resultados}

Del análisis estadístico realizado, cabe destacar los siguientes resultados:

En relación a la hipótesis 1 sobre la mayor preocupación del profesorado por las conductas sociales frente a las conductas instruccionales, se observa que la valoraciones otorgada a hablar y a inhibirse de las tareas son inferiores a las asignadas a las c.sociales, mientras la valoración concedida a desobedecer supera a estas últimas. No se observan diferencias significativas entre la valoración de interrumpir y la valoración de los factores sociales. Ello nos permite afirmar la verificación parcial de nuestra primera hipótesis.

En relación a la hipótesis 2. En primer lugar la comparación los datos según la variable género del profesorado permite obtener los siguientes resultados:

Los profesores no muestran diferencias significativas entre la valoración de las conductas hablar, inhibirse de la tarea e interrumpir y la valoración que hacen de las conductas sociales. No obstante, la valoración de desobedecer es más alta que la valoración de los factores sociales.

Entre las profesoras la valoración de hablar y la de inhibirse de la tarea es inferior a la valoración otorgada a las conductas sociales, mientras que la valoración de desobedecer es superior a estas últimas. No se observan diferencias significativas entre la valoración atribuida a la conducta interrumpir y las consignadas a las conductas sociales.

Por ello, los resultados obtenidos nos permiten observar una marcada tendencia en el cumplimiento de las expectativas manifestadas en la hipótesis 2.1.

En segundo lugar, comparando las valoraciones otorgadas en función de la variable etapa educativa en la que imparten docencia, podemos señalar:

Que en la etapa educativa de Educación Infantil, la valoración de las conductas hablar, inhibirse de la tarea e interrumpir son inferiores a las otorgadas a las conductas sociales, mientras que la valoración de la conducta desobedecer obtiene puntuaciones superiores a todas las conductas citadas. 


\section{Conductas instruccionales}
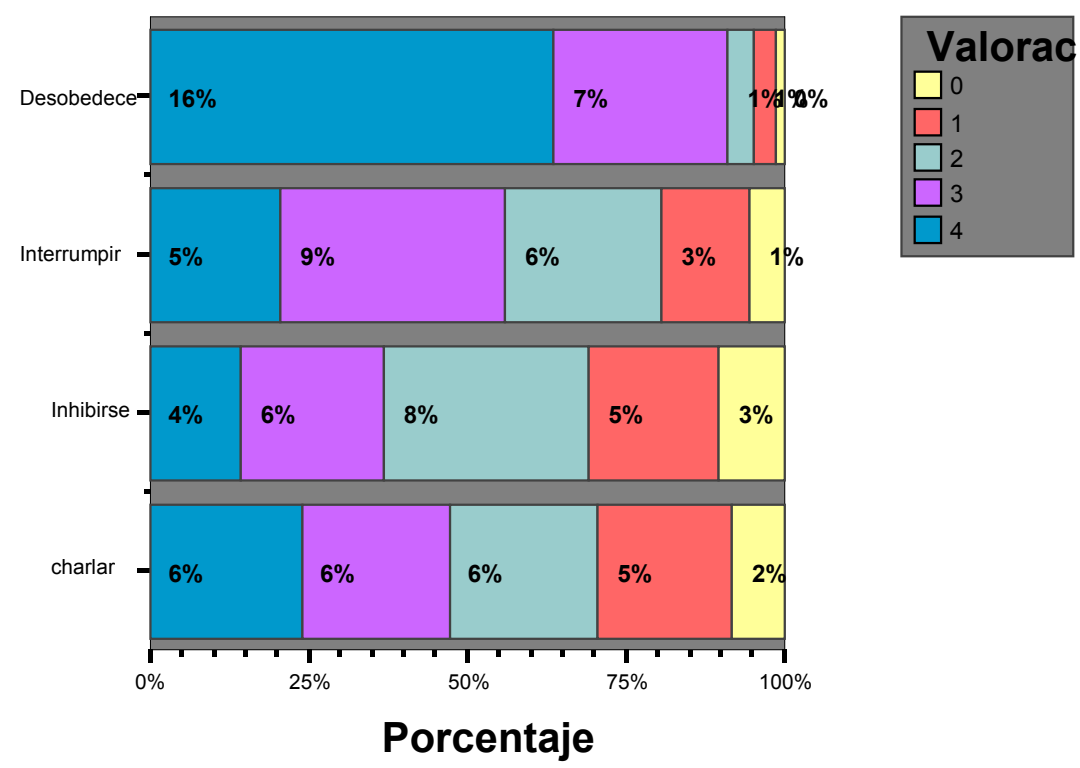

\section{Conductas sociales}

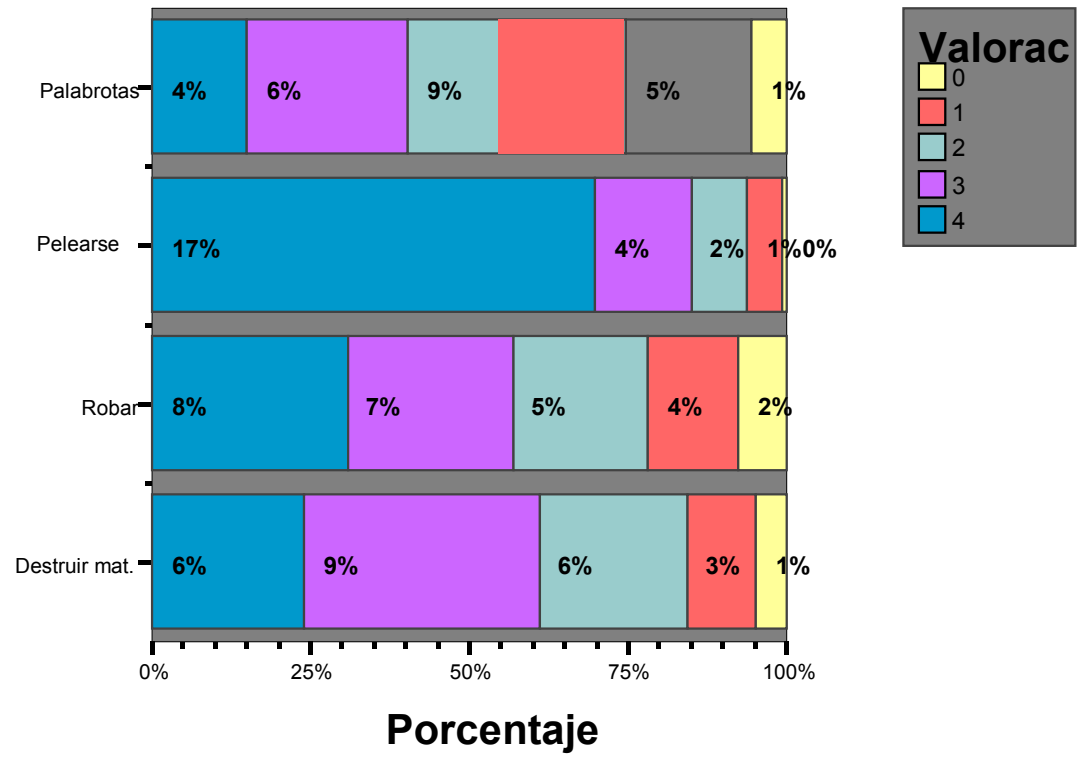

Gráfico 1. Comparación entre valoraciones globales de las conductas instruccionales vs. sociales 


\section{Conductas instruccionales}

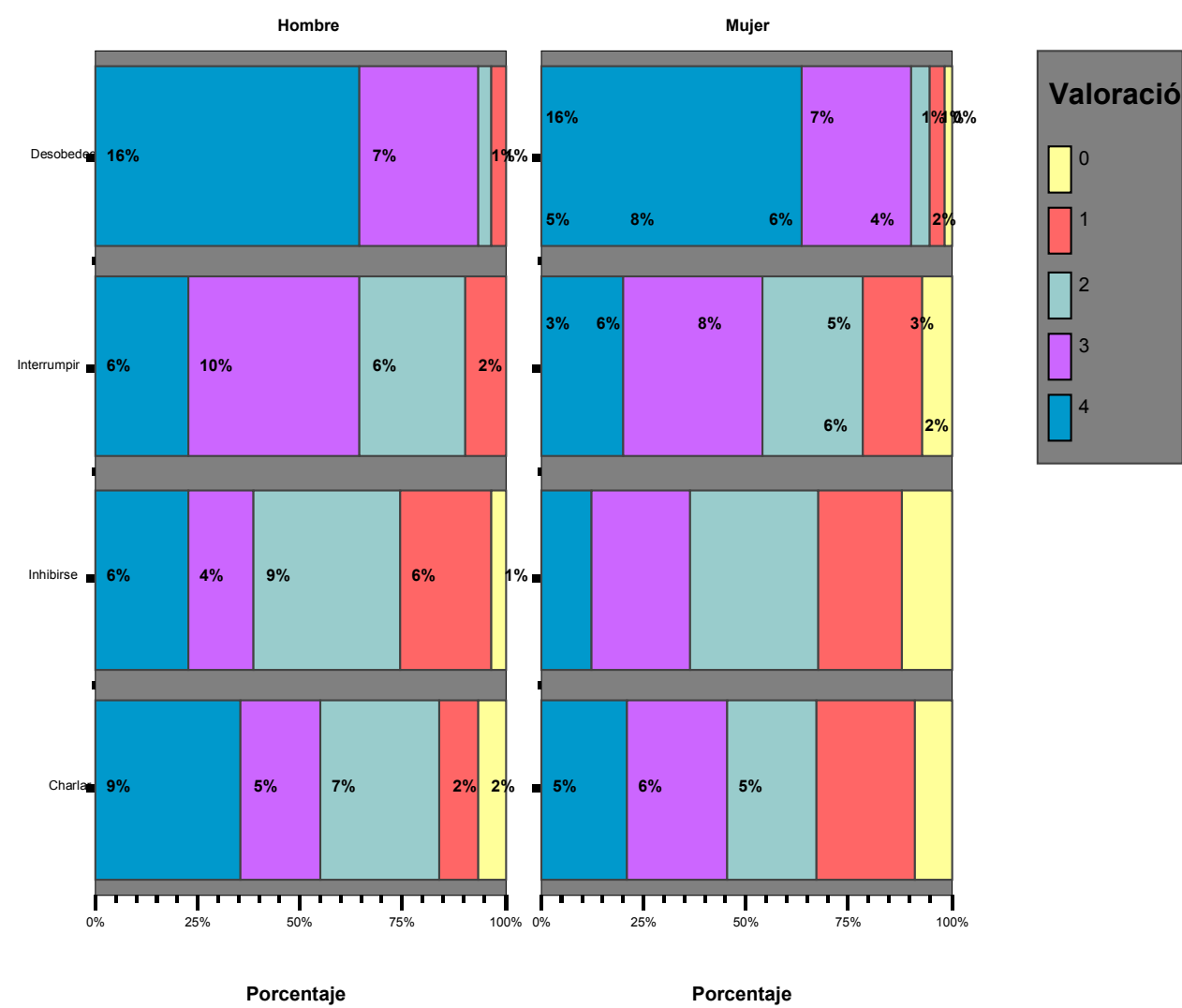

Conductas sociales

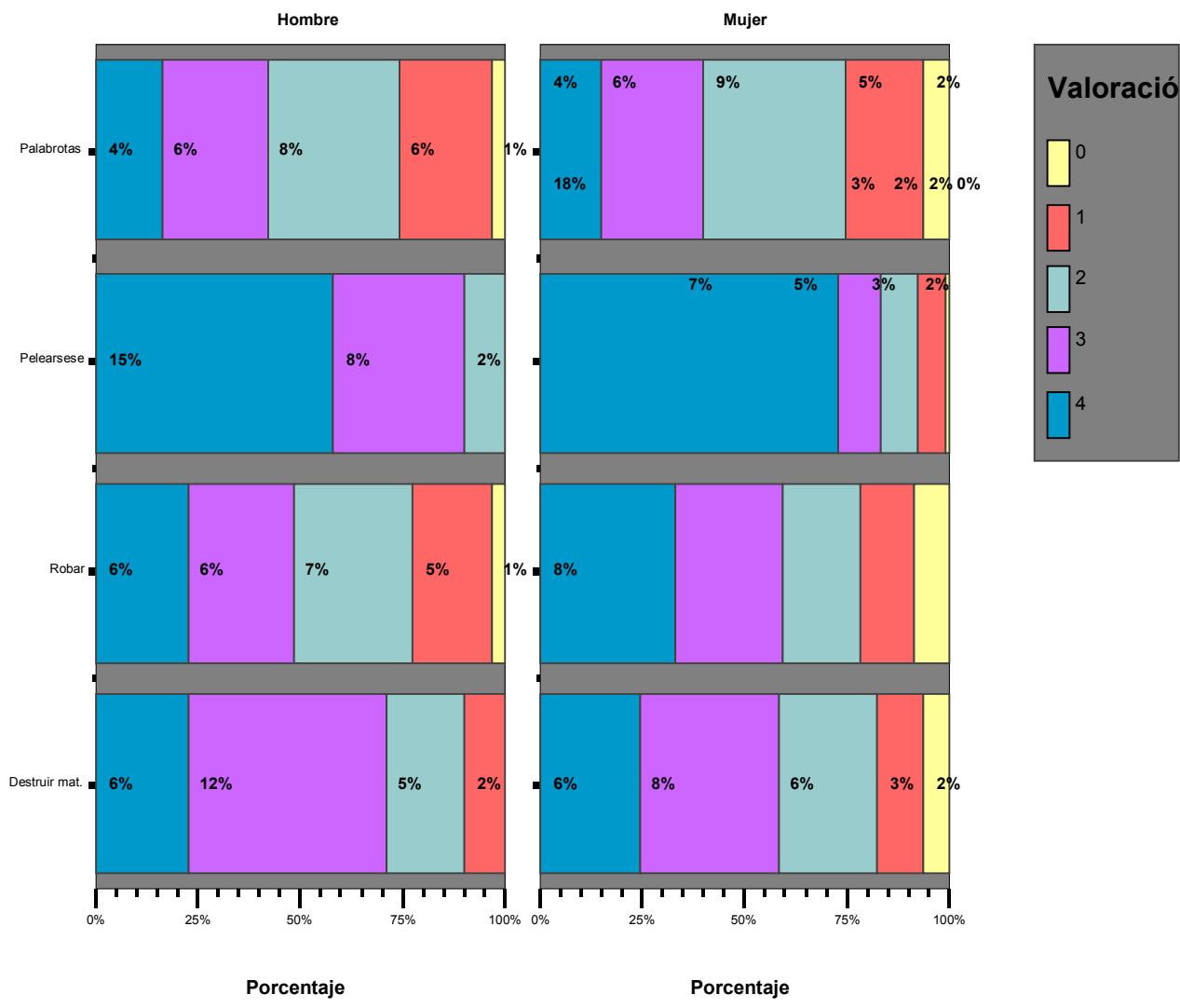

Gráfico 2. Comparación según género 
En la etapa de Educación Primaria, no se observan diferencias significativas entre la valoración de las conductas hablar e interrumpir y la valoración de los factores sociales, pero sí entre la valoración de la conducta inhibirse de la tarea y las correspondientes a las c.sociales, donde la primera recibe puntuaciones inferiores a las segundas, observándose lo contrario en la valoración de la conducta desobedecer que recibe puntuaciones superiores a las atribuidas a las conductas sociales.

En la Etapa de Educación Secundaria Obligatoria, no se observan diferencias significativas entre la valoración de las conductas hablar e interrumpir y la valoración otorgada a las conductas sociales. Frente a ello, la valoración del comportamiento inhibirse de la tarea es inferior y el de desobedecer superior a las puntuaciones atribuidas a las conductas sociales.

Estos resultados son compatibles con la tendencia de nuestras expectativas, si bien no permiten una verificación total de la subhipótesis 2.2 .

En tercer lugar el contraste de los datos según la variable años de experiencia evidencia que:

El profesorado con experiencia igual o inferior a cinco años, valoran las conductas hablar e inhibirse de la tarea e interrumpir con puntuaciones inferiores a las que atribuyen a las conductas sociales. Lo contrario sucede con el comportamiento desobedecer.

Dicho patrón de valoraciones se reproduce, aunque con distinta intensidad, en las respuestas del profesorado con niveles de experiencia entre 6 y 20 años; las valoraciones que otorgan a las conductas hablar, interrumpir e inhibirse de la tarea son significativamente inferiores a la valoración atribuida a las conductas sociales. No obstante, el comportamiento desobedecer se percibe más grave que las conductas sociales.

El profesorado con más de 20 años de experiencia no muestran diferencias significativas entre las valoraciones que atribuyen a las conductas hablar, inhibirse de la tarea e interrumpir y las que otorgan a las conductas sociales. También en este colectivo la valoración atribuida a desobedecer es superior a la otorgada a las conductas sociales.

En definitiva, los resultados indican que se observa una tendencia a atribuir mayor gravedad a los comportamientos instruccionales a medida que aumenta los años de experien- 
cia de los docentes, resultado compatible con las expectativas que plasmadas en la hipótesis 2.3 .

\section{Conductas instruccionales}

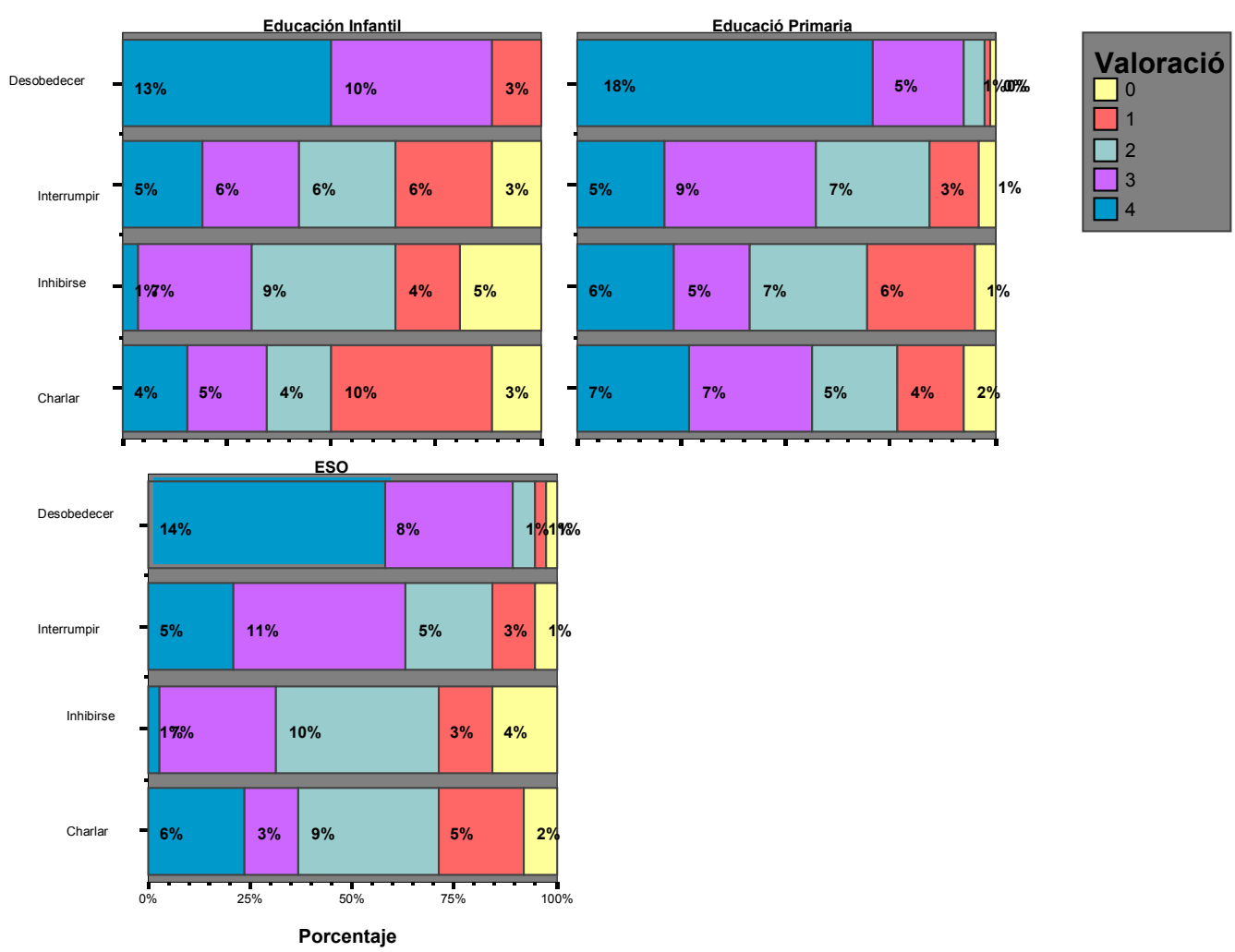

Conductas sociales

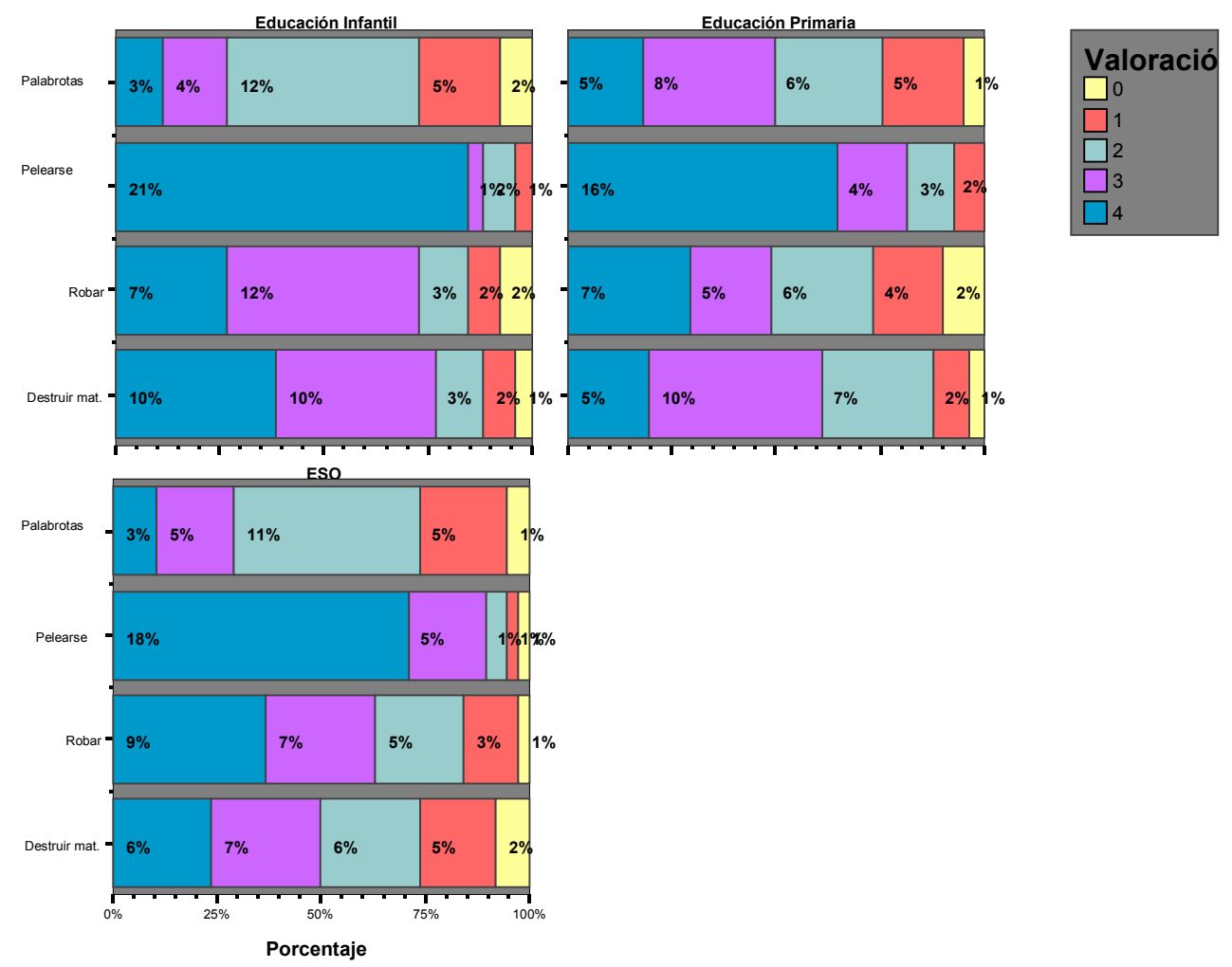

Gráfico 3. Comparación según etapa educativa 


\section{Conductas instruccionales}
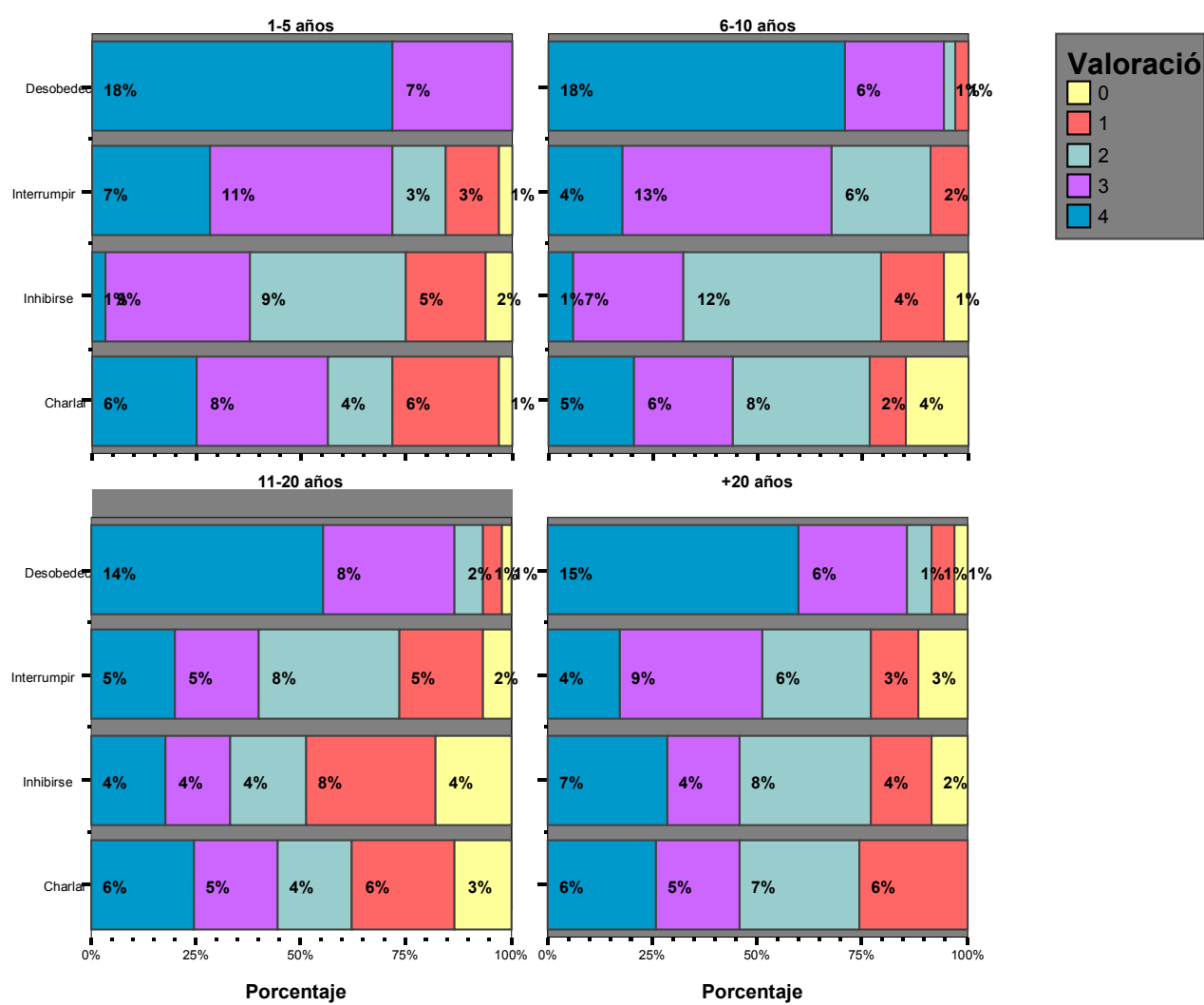

Conductas sociales
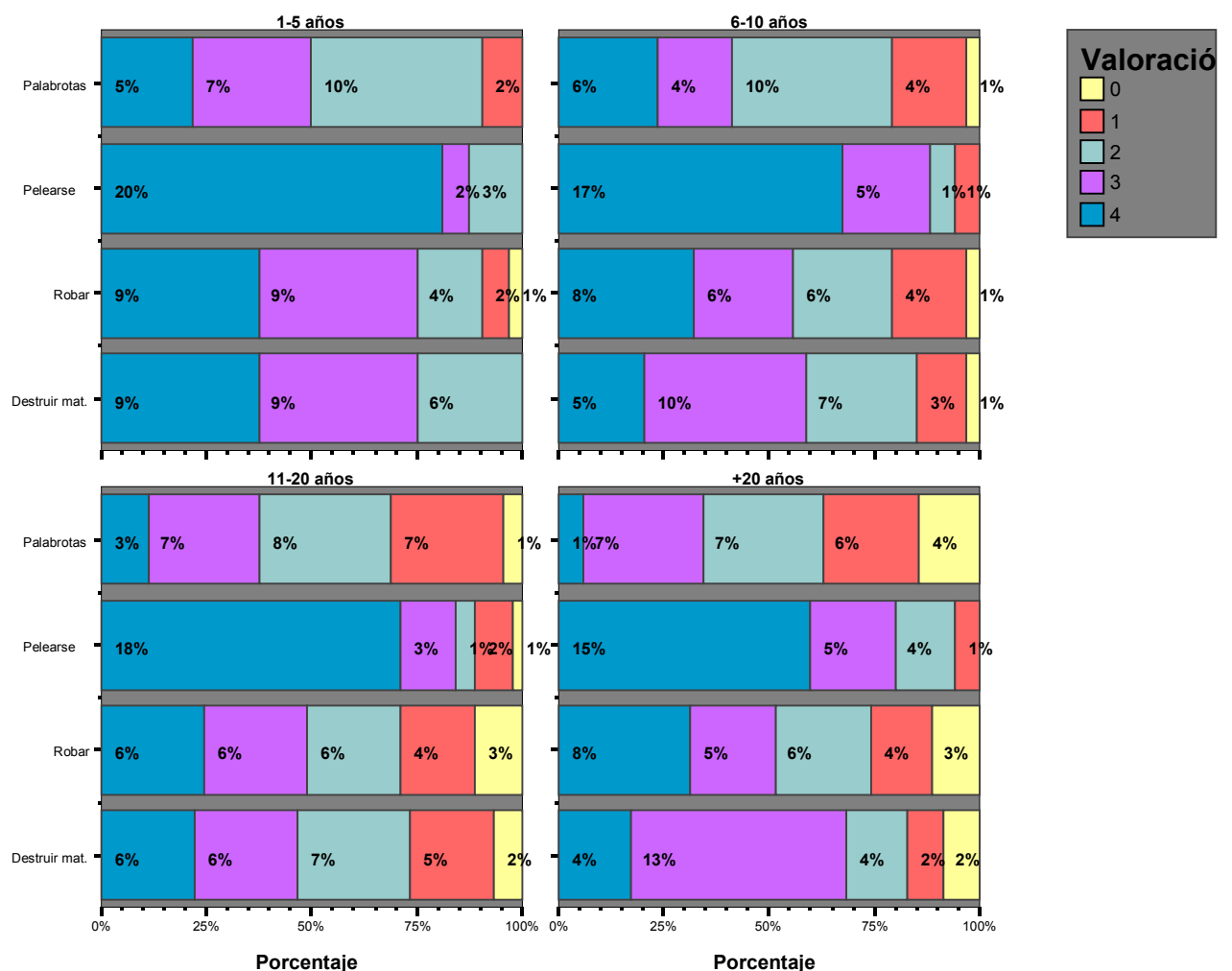

Gráfico 4. Comparación según experiencia. 
Finalmente, si comparamos las valoraciones de los comportamientos instruccionales con la valoración de los factores sociales, teniendo en cuenta la variable población de la muestra, observamos que:

La muestra de la ciudad de Barcelona nos indica que la valoración de las conductas hablar e inhibirse de la tarea son más bajas que la valoración que se hace de las conductas sociales, mientras que la valoración del comportamiento de desobedecer es superior. No obstante, no se observan diferencias significativas entre la valoración de la conducta de interrumpir y la valoración de las conductas sociales.

Respecto a la muestra de la ciudad de Coimbra la valoración de las conductas hablar, inhibirse de la tarea e interrumpir son más bajas que la valoración que se hace de las conductas sociales. No obstante, la valoración del comportamiento de desobedecer es más alta que la valoración atribuida a las conductas sociales.

La muestra de la ciudad de Murcia pone de manifiesto que la valoración de comportamientos tales como hablar, inhibirse y desobedecer son más altas que la valoración otorgada a las conductas sociales.

En este caso, merece destacarse que la muestra de la ciudad de Murcia y a diferencia de lo que se observa en los otros dos grupos de profesores (Barcelona y Coimbra) otorga puntuaciones superiores a la mayoría de las conductas instruccionales que a las conductas sociales. 


\section{Conductas instruccionales}

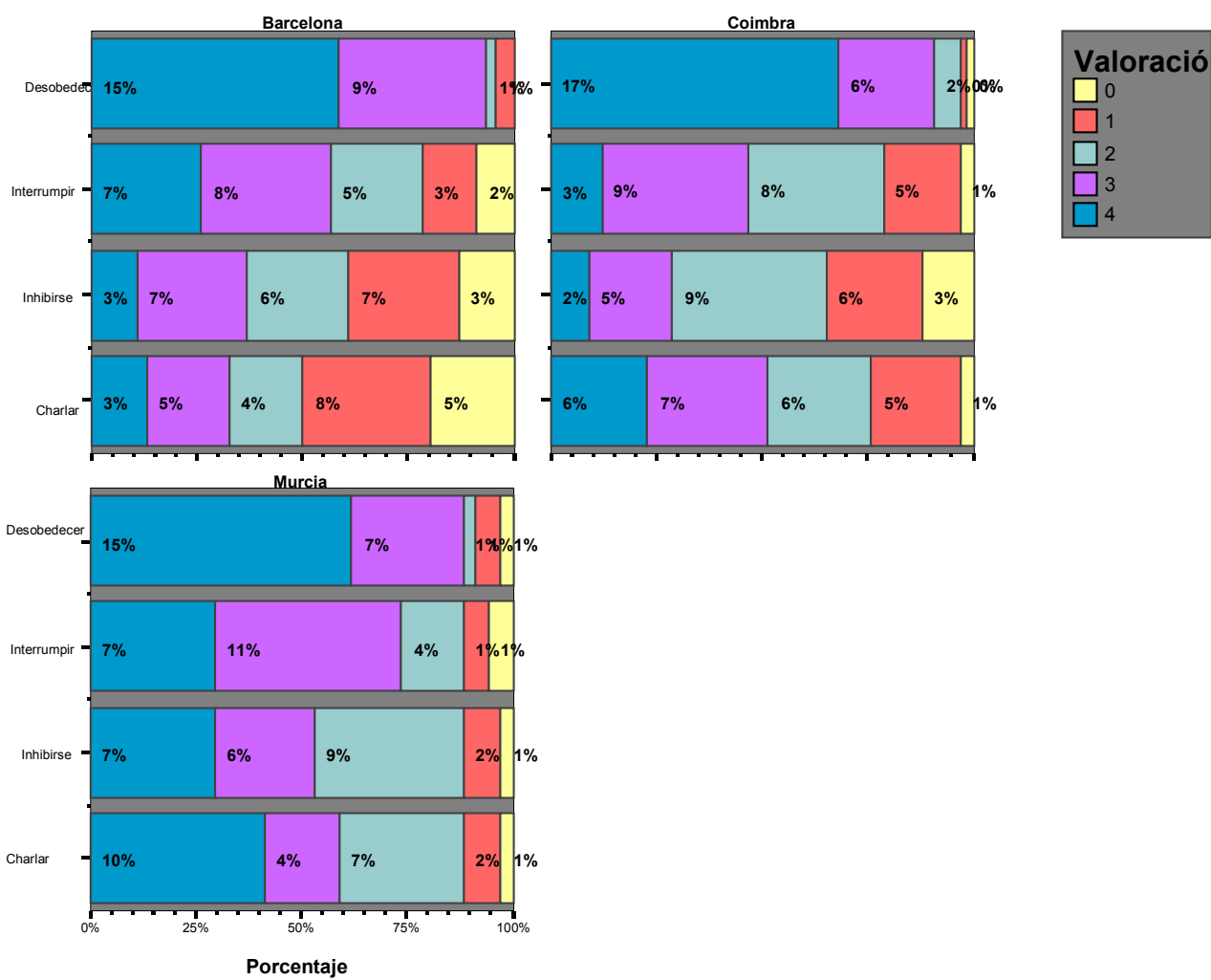

Conductas sociales

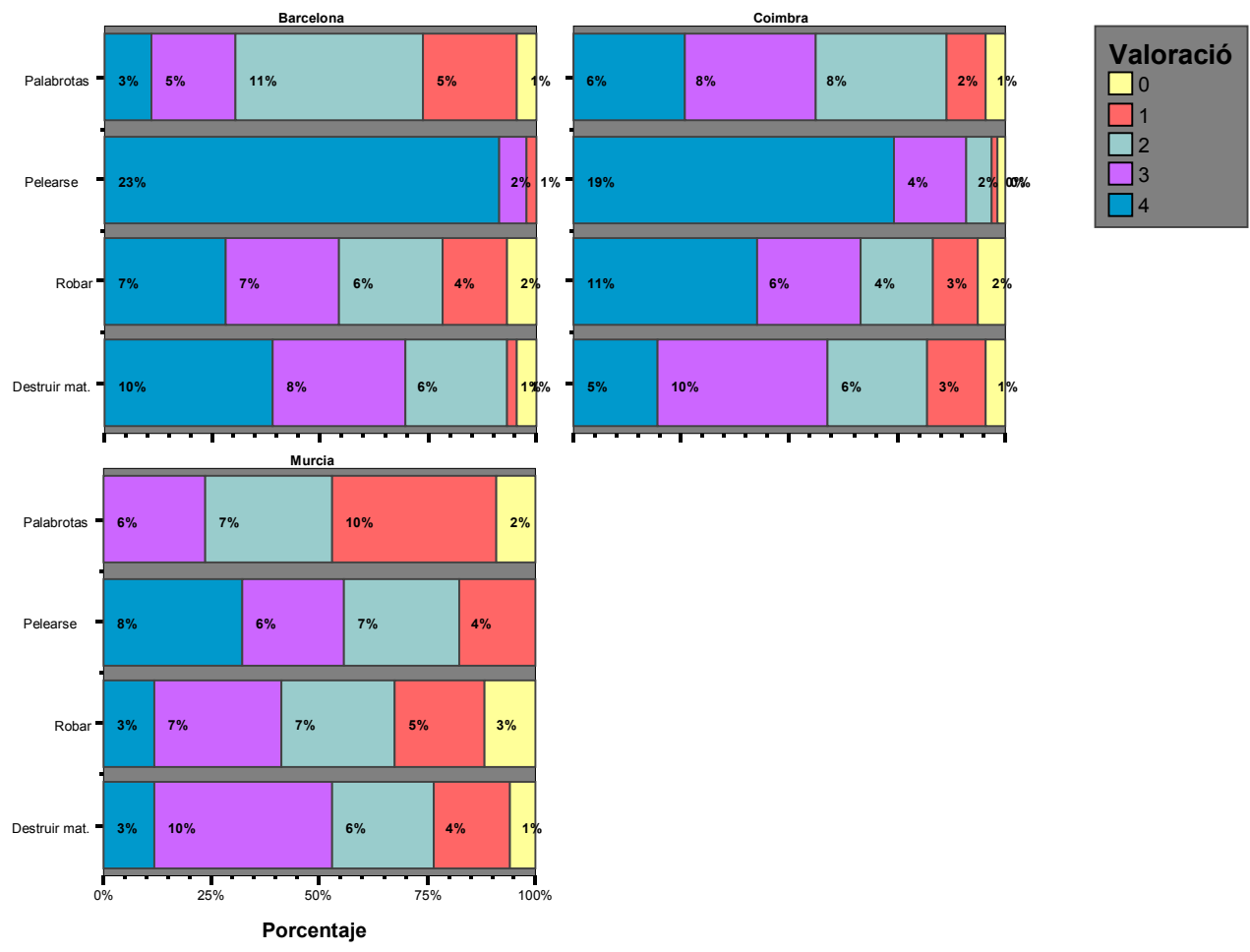

Gráfico 5. Comparación según poblaciones 


\section{Discusión y Conclusiones}

La primera conclusión a destacar es la verificación de nuestra primera hipótesis en tanto se confirma que, aunque el profesorado muestra preocupación por los todas las conductas presentados en el cuestionario, en términos generales otorgan mayor capacidad disruptiva a las "conductas sociales" que a las "conductas intruccionales".

Por una parte, hay que resaltar que todos los comportamientos presentados en este estudio se perciben como fuentes de disrupción en el aula y, por tanto, como amenazas al proceso de enseñanza-aprendizaje, justificando así la intensa preocupación que el tema disciplina escolar entraña. Por otra parte, la verificación de la hipótesis 1 es congruente con la existencia de cierta preocupación descontextualizada por parte del profesorado en lo referente a los comportamientos que poseen mayor poder disruptivo en el aula, ya que otorgan especial atención a comportamientos que no son precisamente los más perjudiciales para la gestión del aula.

Dicha valoración se puede interpretar en el sentido de que los docentes no sólo consideran que las conductas sociales perjudican más el orden en el aula que las denominadas conductas instruccionales, sino que acciones como hablar quedan relegadas a los últimos puestos del ranking, olvidando el papel fundamental que los procesos atencionales desempeñan en los aprendizajes.

Algo semejante cabe decir de inhibirse de la tarea, pues tratándose de una conducta incompatible con el aprendizaje -un alumno ajeno a las tareas de clase difícilmente aprenderá- ocupa los lugares más bajos en el ranking de gravedad atribuida por el profesorado. Sólo la conducta desobedecer ocupa lugares adelantados en el mencionado ranking, mientras que interrumpir, comparte "espacios" con las conductas sociales.

De forma análoga a los resultados obtenidos en otros trabajos ya citados el profesorado muestra mayor preocupación por las transgresiones al orden social general (robar) y por las que resultan perturbadoras para el conjunto del grupo de clase, relegando a posiciones de menor importancia las conductas cuya implicación con los procesos de enseñanza-aprendizaje está rigurosamente comprobada, pero no se asocian con el caos en el aula. 
Estos resultados son inquietantes pues, una vez más, ponen de manifiesto que, a falta de una formación teórica y aplicada sobre la disciplina escolar, el profesorado opta por utilizar el conocimiento creencial para gestionar el orden en sus aulas, de manera que sus decisiones al respecto no se basan tanto en los principios de la enseñanza-aprendizaje cuanto en los de la convivencia social general.

Por cuanto se refiere a las comprobaciones relativas a la hipótesis 2 y sus correspondientes subhipótesis interesa señalar que a pesar de no verificarse de manera íntegra ninguna de ellas, en todos los casos hemos conseguido constatar tendencias en el sentido que marcan nuestras expectativas. Así por ejemplo, podemos afirmar que los años de experiencia parecen ejercer un efecto moderador en la preocupación por las conductas sociales llegándolas a equiparar con la gravedad atribuida a las conductas instruccionales y que dicha equiparación se observa preferentemente entre los docentes del sexo masculino que entre sus compañeras del sexo femenino. En lo que a nivel de enseñanza se refiere, el profesorado de Educación Infantil tiende a priorizar las conductas sociales sobre las instruccionales, en tanto sus colegas de Educación Secundaria otorgan a unos y a otros niveles de gravedad similares.

Sin embargo, tres cuestiones merecen comentario a parte: de un lado, la constatación de que la conducta desobedecer es, en todos los casos, la más valorada; de otro, la valoración sistemáticamente inferior que reciben las conductas hablar e inhibirse de la tarea y finalmente, el hecho de que la submuestra compuesta por los docentes de Murcia es la única que prioriza las conductas instruccionales por encima de las sociales como fuentes de perturbación en el aula.

Será objeto de futuros estudios ofrecer una interpretación cabal a dichos resultados especialmente en lo tocante a las conductas desobedecer, hablar e inhibirse de la tarea pues ya hemos declarado el carácter exploratorio de la comparación entre submuestras geográficas. Por el momento y a la luz de resultados obtenidos en otras investigaciones de este equipo, podemos señalar que la desobediencia del alumnado significa negar o, en el mejor de los casos ignorar, las disposiciones del profesorado lo que hace inviable el desarrollo de un proceso tan complejo como lo es el de enseñar-aprender, en el que la negativa de una de las partes a implicarse en el mismo lo condena indefectiblemente al fracaso. 
Es posible, que la anticipación de estas consecuencias por parte del profesor justifique la preocupación que, ante esta amenaza, muestran los docentes y, por ende, la puntuación que en nuestro cuestionario le otorgan.

En lo que respecta a la conducta inhibirse de la tarea la justificación más pausible es la escasa molestia que genera en el grupo de clase la falta de implicación de alguno de sus miembros. Capítulo a parte merece la tendencia a hablar entre los alumnos continuamente pues su potencial disruptivo en todo proceso de enseñanza-aprendizaje es elevado; tal vez se trate de un comportamiento tan extendido que no se perciba como grave, sin olvidar que han sido muchas las voces que se han alzado en defensa del comportamiento libre y espontáneo del alumnado, lo que resultaría compatible con la permisividad ante estas formas de expresión.

En todo caso, lo que se pone de manifiesto es que los docentes no disponen de una conceptualización nítida y completa de las conductas que poseen mayor potencial disruptivo en las aulas que no son otras que las más alteran los procesos de aprendizaje que, individual y colectivamente, lleva a cabo el alumnado. 


\section{Referencias}

Añaños, E. y Gotzens, C. (1990). Análisis de algunos elementos que intervienen en la conducta del profesor: un estudio a partir de la resolución de problemas de disciplina. Boletín de Psicología, 2, 77-93.

Badia, M (2001). Las percepciones de profesores y alumnos de E.S.O sobre la intervención en el comportamiento disruptivo: un estudio comparativo. Tesis Doctoral. Bellaterra (Barcelona). Universidad Autónoma de Barcelona.

Benítez, J.L., García-Berbén, A. y Fernández, Ma . (2009). Impacto de un curso sobre maltrato entre iguales en el currículum universitario del profesorado. Electronic Journal of Research in Educational Psychology, 17, 7(1), 191-208.

Bibou N.I., Kiosseoglou, G. y Stogiannidou, A. (2000). Elementary teachers' perceptions regarding school behavior problems: Implications for school psychological services. Psychology in the Schools, 37(2), 123-134.

Borko, H., Lalik, R. y Tomchin, E. (1987): Students Teachers' Understandings of Successful and Unsuccessful Teaching. Teaching and Teacher Education, 3, 77-90.

Calderhead, J. (1996). Teachers: beliefs and Knowledge. En D.C. Berliner y R. Calfee (Eds). Handbook of Educational Psychology, (709-725). New York. Macmillan.

Castelló, A. (1999). Procesos cognitivos en el professor. En A. Sipán (Coord.). Educar para la diversidad en el S.XXI, 81-107. Zaragoza. Mira Editores.

Corrie, L. (1997). The interaction between teachers'knowledge and skills when managing a troublesome classroom behaviour. Cambridge Journal of Education, 27(1), 93-105.

Fernández-Balboa, J. M. (1990). Helping Novice Teachers Handle Discipline Problems Journal of Physical Education, Recreation, and Dance, 62, 50-54.

Fernández-Balboa, J. M (1991). Beliefs, Interactive Thoughts, and Actions ff Physical Education Student Teachers Regarding Pupil Misbehaviors. Journal of Teaching in Physical Education, 11, 59-78.

Genovard, C. y Gotzens, C. (1990). Psicología de la Instrucción. Madrid. Santillana.

Gotzens, C. (1985) La perspectiva dels adults sobre els comportaments d'indisciplina en el context escolar. En VVAA Psicopedagogia de l'excepcionalitat: Barcelona: Fundació Caixa de Pensions.

Gotzens, C. (1997). La disciplina escolar. Horsori. Barcelona.

Gotzens, C. (2006). El psicólogo escolar: nuevos retos y viejos desencuentros. Papeles del Psicólogo, 27, 180-184. 
Gotzens, C., Badia, M., Castelló, A. y Genovard, C. (2007). La gravedad de los problemas de comportamiento en el aula vista por los profesores. Revista Portuguesa de Pedagogía, 4l(1), 103-120.

Gotzens, C., Castelló, A., Genovard, C. y Badia, M. (2003). Percepciones de profesores y alumnos de E.S.O. sobre la disciplina en el aula. Psicothema, 15(3), 362-368.

Grebennikov, L. (2005). The Normalised Child: A Non-Traditional Psychological Framework. Australian Journal of Early Childhood, 30(2), 8-14.

Gump, P.V. (1980). The school as a social situation. Annual Review of Psychology, 31, 553582.

Hardman, E.L. y Smith, S. (2003). Analysis of Classroom Discipline-Related Content in Elementary Education Journals. Behavioral Disorders. 28(2), 173-186.

Houhton, S., Merrett, F. y Whendall, K. (1988). Classroom behaviours which secondary schools teachers say they find most troublesome. British Educational Research Journal, 14, 295-310.

Ishee, J. H. (2004), Perceptions of Misbehavior in Middle School Physical Education. Journal of Physical Education, Recreation and Dance, 75, 9.

Kounin J.S (1977). Discipline and group management in classrooms. New York: Holt, Rinehart \& Winston.

McLeod, J., Fisher, J. y Hoover, G. (2003). The Key Elements of Classroom Management: Managing Time and Space, Student Behavior, and Instructional Strategies. Alexandria, VA: Association for Supervision and Curriculum Development.

Meunier, J.M. (2000). Sondage dans une ecole primaire: Des conduites pacifiques et des comportements violents. Apprentissage et Socialisation, 20(1), 35-49.

Mumby, H., Russell, T. y Martin, A.K. (2001). Teachers'knowledge and how it develops. En V. Richardson (Ed.). Handbook of Research on Teaching (4 ${ }^{\text {th }}$ edition) 119-160. Washington: American Educational Research Association.

Pajares, M.F. (1992). Teachers' beliefs and educational research: Clearing up a construct. Review of educational Research, 62, 307-332.

Ramírez, S. y Justicia, F. (2006). El maltrato entre escolares y otras conductas-problema para la convivencia. Electronic Journal of Research in Educational Psychology 9, 4(2), 265-290.

Richmond, V.P. y McCroskey, J.C. (Eds.) (1992). Power in the Classroom: Communication, Control, and Concern. Hillsdale, NJ: Lawrence Erlbaum Associates. 
Rudduck, J., Chaplain, R. y Wallace, G. (1996). Reviewing the conditions of learning in school, En J. Rudduck, R. Chaplain y G. Wallace (Eds). School Improvement: What can pupil tell us? 29-40. London: David Fulton Publishers.

Seidman, A. (2005). The Learning Killer: Disruptive student behaviour in the classroom. Reading improvement, 42(1), 40-49.

Slavin, R.E (1989). School and Classroom Organization. Hillsdale, NJ: Lawrence Erlbaum Associates.

Watkins, CH. y Wagner, P. (1991) La disciplina escolar. Propuesta de trabajo en el marco global del centro. Barcelona: Paidós.

White, R., Algozzine, B., Audette, R., Marr, M. B., y Ellis, E. D. (2001). Unified Discipline: A School-Wide Approach for Managing Problem Behavior. Intervention in School \& Clinic, 37(1), 3-11.

Wickman, E.K. (1928). Children's behavior and teachers attitudes. New York: The Commonwealth Fundation.

Woolfolf, A., Rarosoff, R. y Hoy, W. (1998). Teacher's sense of efficacy and their beliefs about managing students. Teaching and Teacher Education. 6(2), 46-54. 